\title{
RADIAL SOLUTIONS AND PHASE SEPARATION IN A SYSTEM OF TWO COUPLED SCHRÖDINGER EQUATIONS
}

\author{
JUNCHENG WEI AND TOBIAS WETH
}

ABStRact. We consider the nonlinear elliptic system

$$
\begin{cases}-\Delta u+u-u^{3}-\beta v^{2} u=0 & \text { in } \mathbb{B}, \\ -\Delta v+v-v^{3}-\beta u^{2} v=0 & \text { in } \mathbb{B}, \\ u, v>0 \quad \text { in } \mathbb{B}, \quad u=v=0 & \text { on } \partial \mathbb{B},\end{cases}
$$

where $N \leq 3$ and $\mathbb{B} \subset \mathbb{R}^{N}$ is the unit ball. We show that, for every $\beta \leq-1$ and $k \in \mathbb{N}$, the above problem admits a radially symmetric solution $\left(u_{\beta}, v_{\beta}\right)$ such that $u_{\beta}-v_{\beta}$ changes sign precisely $k$ times in the radial variable. Furthermore, as $\beta \rightarrow-\infty$, after passing to a subsequence, $u_{\beta} \rightarrow w^{+}$and $v_{\beta} \rightarrow w^{-}$uniformly in $\mathbb{B}$, where $w=w^{+}-w^{-}$has precisely $k$ nodal domains and is a radially symmetric solution of the scalar equation $\Delta w-w+w^{3}=0$ in $\mathbb{B}, w=0$ on $\partial \mathbb{B}$. Within a Hartree-Fock approximation, the result provides a theoretical indication of phase separation into many nodal domains for Bose-Einstein double condensates with strong repulsion.

\section{INTRODUCTION}

The present paper is concerned with the study of solitary wave solutions for the coupled Gross-Pitaevskii equations

$$
\left\{\begin{array}{l}
-i \frac{\partial}{\partial t} \Phi_{1}=\Delta \Phi_{1}+\mu_{1}\left|\Phi_{1}\right|^{2} \Phi_{1}+\beta\left|\Phi_{2}\right|^{2} \Phi_{1} \text { for } y \in \Omega, t>0 \\
-i \frac{\partial}{\partial t} \Phi_{2}=\Delta \Phi_{2}+\mu_{2}\left|\Phi_{2}\right|^{2} \Phi_{2}+\beta\left|\Phi_{1}\right|^{2} \Phi_{2} \text { for } y \in \Omega, t>0 \\
\Phi_{1}(y, t)=\Phi_{2}(y, t)=0 \text { for } y \in \partial \Omega, t>0
\end{array}\right.
$$

where $\mu_{1}, \mu_{2}$ are positive constants, $\Omega$ is a domain in $\mathbb{R}^{N}, N \leq 3$, and $\beta$ is a coupling constant. System (1.1) arises in the Hartree-Fock theory for a double condensate, i.e., a binary mixture of Bose-Einstein condensates in two different hyperfine states $|1\rangle$ and $|2\rangle$, see [15]. Physically, $\Phi_{1}$ and $\Phi_{2}$ are the corresponding condensate amplitudes, $\mu_{1}$ and $\mu_{2}$ are proportional to the intraspecies scattering lengths, and $\beta$ is proportional to the interspecies scattering length. The sign of $\mu_{j}$ determines whether collisions of particles of the single state $|j\rangle$ result in a repulsive or attractive interaction, while the sign of $\beta$ determines the interaction of particles of state $|1\rangle$ and state $|2\rangle$. If $\mu_{j}>0$ as considered here, we are dealing with an attractive self-interaction of the single states $|j\rangle, j=1,2$. When $\beta<0$, the interaction of state $|1\rangle$ and $|2\rangle$ is repulsive (as discussed in [37]). In contrast, when $\beta>0$, the interaction of state $|1\rangle$ and $|2\rangle$ is attractive. 
When $\Omega=\mathbb{R}^{N}$, system (1.1) also arises in the study of incoherent solitons in nonlinear optics. We refer to $[27,28]$ for experimental results and to $[3,9,19-21]$ for a comprehensive list of references.

To obtain solitary wave solutions of the system (1.1), we set $\Phi_{1}(x, t)=e^{i \lambda_{1} t} u(x)$, $\Phi_{2}(x, t)=e^{i \lambda_{2} t} v(x)$, and the system (1.1) is transformed to an elliptic system given by

$$
\left\{\begin{array}{cl}
-\Delta u+\lambda_{1} u-\mu_{1} u^{3}-\beta v^{2} u=0 & \text { in } \Omega, \\
-\Delta v+\lambda_{2} v-\mu_{2} v^{3}-\beta u^{2} v=0 & \text { in } \Omega, \\
u, v>0 \quad \text { in } \Omega, \quad u=v=0 & \text { on } \partial \Omega .
\end{array}\right.
$$

As shown by recent results, the structure of the solution set of (1.2) depends strongly on the value of $\beta$. For a bounded domain $\Omega \subset \mathbb{R}^{N}, N \leq 3$, a least energy solution of (1.2) exists within the range $\beta \in\left(-\infty, \beta_{0}\right]$, where $0<\beta_{0}<\sqrt{\mu_{1}, \mu_{2}}$ is a constant. This is proved in [23], where also the asymptotic behavior of this solution is studied as the domain $\Omega$ becomes large. When $\Omega=\mathbb{R}^{N}$, the existence of least energy and other finite energy solutions of (1.2) is proved in $[2,5,25,35]$ for $\beta$ belonging to different subintervals of $(0, \infty)$. It is important to note that when $\Omega$ is a ball or $\Omega=\mathbb{R}^{N}$ and $\beta>0$, then all solutions of (1.2) are radially symmetric (up to translation if $\Omega=\mathbb{R}^{N}$ ), and both components are decreasing in the radial variable, see [38]. In contrast, different classes of nonradial solutions, distinguished by their shape and symmetries, have been constructed for $\Omega=\mathbb{R}^{N}$ and $\beta<0,|\beta|$ small in [24] and for $\beta \leq-1$ in [43]. In the present paper we analyze another class of solutions of (1.2) which only exist for negative $\beta$, namely radial but not radially decreasing solutions when $\Omega=\mathbb{B}$ is the unit ball in $\mathbb{R}^{N}$. We focus on the symmetric case $\lambda_{1}=\lambda_{2}, \mu_{1}=\mu_{2}$, assuming without loss of generality that $\lambda_{1}=\lambda_{2}=\mu_{1}=\mu_{2}=1$. Hence we study radial solutions of the following nonlinear elliptic system:

$$
\begin{cases}-\Delta u+u-u^{3}-\beta v^{2} u=0 & \text { in } \mathbb{B}, \\ -\Delta v+v-v^{3}-\beta u^{2} v=0 & \text { in } \mathbb{B}, \\ u, v>0 \quad \text { in } \mathbb{B}, \quad u=v=0 & \text { on } \partial \mathbb{B} .\end{cases}
$$

Our results establish a connection between radial solutions of (1.3) and sign changing radial solutions of the scalar problem

$$
-\Delta w+w-w^{3}=0 \quad \text { in } \mathbb{B}, \quad w=0 \quad \text { on } \partial \mathbb{B} .
$$

Let $H_{r}$ be the Hilbert space of all radially symmetric functions in $H_{0}^{1}(\mathbb{B})$ endowed with the norm $\|u\|^{2}:=\int_{\mathbb{B}}\left(|\nabla u|^{2}+|u|^{2}\right) d x$. Radial solutions of (1.3) are critical points of the energy functional $E: H_{r} \times H_{r} \rightarrow \mathbb{R}$ given by

$$
E(u, v)=\frac{1}{2}\left(\|u\|^{2}+\|v\|^{2}\right)-\frac{1}{4} \int\left(u^{4}+v^{4}\right) d x-\frac{\beta}{2} \int u^{2} v^{2} d x,
$$

Moreover, radial solutions of (1.4) are critical points of the functional

$$
E_{S}: H_{r} \rightarrow \mathbb{R}, \quad E_{S}(w)=\frac{1}{2}\|w\|^{2}-\frac{1}{4} \int w^{4} d x .
$$


To state our main results, we recall that, for every $k \in \mathbb{N}$, (1.4) admits a radial solution with precisely $k$ nodal domains, i.e., $k-1$ sign changes in the radial variable, see $[40,41]$. In dimension $N=1$ this solution is unique (see [39]), but for $N>1$ this is unknown. We put

$$
c_{k}:=\inf _{w \in \mathcal{S}_{k}} E_{S}(w), \quad(k \in \mathbb{N}),
$$

where $\mathcal{S}_{k} \subset H_{r}$ is the set of radial solutions of (1.4) with precisely $k$ nodal domains. There exists a different characterization of $c_{k}$ via a variational principle introduced by Nehari [30], see Proposition 2.1 below. Our first main result is the following.

Theorem 1.1. Let $N \leq 3$. Then for every $\beta \leq-1$ and every integer $k \geq 2$, (1.3) admits a solution $(u, v) \in H_{r} \times H_{r}$ such that $E(u, v) \leq c_{k}$ and $u-v$ changes sign precisely $k-1$ times in the radial variable.

Theorem 1.1 yields the existence of infinitely many radial solutions $(u, v)$ of $(1.3)$ which are distinguished by the number of intersections of $u$ and $v$. For fixed $k$, these solutions satisfy an energy bound independent of the coupling parameter $\beta$. Our second main result provides a description of the limit shape of these solutions as $\beta$ tends to minus infinity.

Theorem 1.2. Let $N \leq 3, k \geq 2$, and let $\beta_{n} \leq-1, n \in \mathbb{N}$ be a sequence of numbers with $\beta_{n} \rightarrow-\infty$ as $n \rightarrow \infty$. Let also $\left(u_{n}, v_{n}\right) \in H_{r} \times H_{r}$ be solutions of (1.3) with $\beta=\beta_{n}$ such that $u_{n}-v_{n}$ changes sign precisely $k-1$ times (in the radial variable) and $E\left(u_{n}, v_{n}\right) \leq c_{k}$.

Then, after passing to a subsequence, $u_{n} \rightarrow w^{+}$and $v_{n} \rightarrow w^{-}$in $H_{r}$ and $C(\overline{\mathbb{B}})$, where $w$ is a solution of (1.4) with precisely $k-1$ interior zeros and $E(w)=c_{k}$.

Here and in the following, $w^{+}=\max \{w, 0\}$ and $w^{-}=-\min \{w, 0\}$ denote the positive and negative part of a function $w: \mathbb{B} \rightarrow \mathbb{R}$.

In the context of Bose-Einstein condensates (where $\Phi_{1}(x, t)=e^{i t} u(x), \Phi_{2}(x, t)=$ $e^{i t} v(x)$ stand for the amplitudes of the different hyperfine states $|1\rangle$ and $\left.|2\rangle\right)$, the limit shape considered in Theorem 1.2 models the spatial separation of $|1\rangle$ and $|2\rangle$ in the presence of strong repulsion. This phase separation has drawn the attention both from experimental and theoretical physicists [17,29,37], but rigorous mathematical results are rare. In fact, for a general bounded domain $\Omega$ and an arbitrary uniformly bounded solution sequence $\left(u_{\beta}, v_{\beta}\right)$ of $(1.2)$ corresponding to $\beta \rightarrow-\infty$, the corresponding limit profile $(u, v)$, i.e., the weak limit in $\left[H_{0}^{1}(\Omega)\right]^{2}$ of a subsequence, is not well understood. It is easy to see that the nodal sets $N_{u}=\{x \in \Omega: u(x)>0\}$ and $N_{v}=\{x \in \Omega: v(x)>0\}$ are disjoint. Moreover, it is natural to expect that $u$ and $v$ are continuous and therefore $N_{u}$ and $N_{v}$ are open subsets of $\Omega$, but to our knowledge this has not been proved yet. For a related system with different parameter values, Chang-Lin-Lin-Lin [8] proved that $u$ and $v$ solve scalar limit equations in $N_{u}$ and $N_{v}$ under the crucial assumption that $N_{u}, N_{v}$ are open in $\Omega$. Via numerical computations, they investigate further properties of the corresponding nodal domains, i.e., the connected components of $N_{u}$ and $N_{v}$. 
In the radial case, Theorems 1.1 and 1.2 exhibit a large class of solutions which converge uniformly as $\beta \rightarrow-\infty$ and give rise to continuous limit profiles with arbitrarily many nodal domains. Moreover, these limit profiles have matching derivatives of $u$ and $v$ at the common boundary of $N_{u}$ and $N_{v}$.

It is worth pointing out that spatial segregation has been studied already for different classes of competing species systems with simpler coupling terms, see e.g. $[13,14]$. Moreover, the asymptotic behaviour of least energy solutions to a related class of superlinear elliptic systems with strong competition is studied in [12]. In fact, although the nonlinear terms in system (1.2) do not satisfy the growth conditions assumed in [12], it seems that many of the arguments in [12] also apply to least energy solutions of (1.2).

We briefly describe the paper's organisation and the methods used in the proofs. In Section 2 we collect preliminaries on the variational framework for (1.3), and we discuss properties of a parabolic system corresponding to (1.3). A crucial property is the nonincrease of the number of intersections of $u$ and $v$ along trajectories of the associated parabolic semiflow. This nonincrease is an easy consequence of the zero number diminishing property for the scalar problem derived in [32]. In Section 3 we use the parabolic flow, together with a slightly modified version of the classical Krasnoselskii genus, to prove Theorem 1.1. For scalar elliptic equations, special solutions have already been constructed via a corresponding parabolic flow and comparison principles, see $[10,11,33]$. The approach presented here differs from these existing techniques but could also be applied to scalar equations with odd nonlinearities.

Section 4 contains the proof of Theorem 1.2. Here we combine Nehari's variational principle with comparison arguments and ordinary differential equations techniques. In particular, a Ljapunov function for radial solutions of (1.3) is used as a crucial tool to control the number of intersections of $u$ and $v$ while passing to the limit $\beta \rightarrow-\infty$.

We finally remark that it is open whether an existence result similar to Theorem 1.1 also holds for the nonsymmetric system (1.2) in $\Omega=\mathbb{B}$. Since our method uses the genus, it does not apply to (1.2). For a class of superlinear ODE-systems, solutions with a prescribed number of zeroes of each component were constructed in [36] without assuming oddness of the nonlinearity. It is tempting to rewrite system (1.2) in $x=u-v$ and $y=u+v$ in order to apply a similar approach as in [36] to the resulting system. However, even in the symmetric case one obtains a system of the form $-\Delta x+x=\left(\frac{1+\beta}{4}\right) x^{3}+\left(\frac{3-\beta}{4}\right) y^{2} x,-\Delta y+y=\left(\frac{1+\beta}{4}\right) y^{3}+\left(\frac{3-\beta}{4}\right) x^{2} y$, where, for $\beta<-1$, the nonlinear terms have precisely the opposite sign as in (1.3). Therefore this system has completely different properties than the class of systems considered in [36]. Moreover, the condition $u, v>0$ translates into the somewhat unnatural constraint $|x|<y$.

Acknowledgments. The research of the first author is partially supported by an Earmarked Grant from RGC of Hong Kong. Part of the paper was written while the second author was visiting the Chinese University of Hong Kong, to which he is deeply grateful for its hospitality. 


\section{Preliminaries and the CORRESPonding PARABolic PROBlem}

Throughout the remainder of this paper we assume that $N \leq 3$. In this section we consider a fixed coupling constant $\beta \leq-1$ in (1.3). Multiplying the first equation in (1.3) with $u$, the second with $v$ and integrating, we find that all nontrivial solutions of (1.3) are contained in the set

$$
\mathcal{M}=\left\{\begin{array}{l|l}
(u, v) \in H_{0}^{1}(\mathbb{B}) \times H_{0}^{1}(\mathbb{B}), \mid \begin{array}{l}
\|u\|^{2}-\beta|u v|_{2}^{2}=|u|_{4}^{4}, \\
u, v \geq 0, u, v \not \equiv 0
\end{array} \\
\|v\|^{2}-\beta|u v|_{2}^{2}=|v|_{4}^{4}
\end{array}\right\}
$$

Here and in the following, we write $|u|_{p}$ for the usual $L^{p}$-Norm of a function $u \in L^{p}(\mathbb{B})$. We note that

$$
E(u, v)=\frac{1}{4}\left(\|u\|^{2}+\|v\|^{2}\right) \quad \text { for }(u, v) \in \mathcal{M}
$$

Similarly, all nontrivial solutions of (1.4) are contained in

$$
\mathcal{M}_{S}:=\left\{w \in H_{0}^{1}(\mathbb{B}), w \neq 0:\|w\|^{2}=|w|_{4}^{4}\right\},
$$

and $E_{S}(w)=\frac{\|w\|^{2}}{4}$ for $w \in \mathcal{M}_{S}$.

Next, we consider the set $\Gamma_{k} \subset H_{r}$ of all functions $w \in H_{r}$ such that there exists radii $0=r_{0}<r_{1}<\ldots<r_{k-1}<r_{k}=1$ with $w \cdot 1_{\left\{r_{j} \leq|x| \leq r_{j+1}\right\}} \in \mathcal{M}_{S}$ for $j=$ $0, \ldots, k-1$. The following highly useful variational principle goes back to Nehari [30] in the one-dimensional case. Later it was generalized to radial functions in higher space dimensions, see $[6,40,41]$.

Proposition 2.1. The value $c_{k}$ defined in (1.5) admits the variational characterization

$$
c_{k}=\inf _{w \in \Gamma_{k}} E_{S}(w) .
$$

Moreover, if $w \in \Gamma_{k}$ satisfies $E_{S}(w)=c_{k}$ and

$$
\begin{aligned}
& (-1)^{j} w(x) \geq 0 \text { for } r_{j} \leq|x| \leq r_{j+1}, \quad j=0, \ldots, k-1 \quad \text { or } \\
& (-1)^{j} w(x) \leq 0 \text { for } r_{j} \leq|x| \leq r_{j+1}, \quad j=0, \ldots, k-1,
\end{aligned}
$$

then $w$ is a radial solution of (1.4) with precisely $k-1$ interior zeros.

Next we fix $3<p<\infty$, and we consider the function spaces

$$
\begin{aligned}
W_{r} & =\left\{u \in W_{0}^{1, p}(\mathbb{B}): u \text { radially symmetric }\right\}, \\
C_{r} & =\left\{u \in C(\overline{\mathbb{B}}): u \text { radially symmetric, }\left.u\right|_{\partial \mathbb{B}}=0\right\}, \\
C_{r}^{1} & =\left\{u \in C^{1}(\overline{\mathbb{B}}): u \text { radially symmetric, }\left.u\right|_{\partial \mathbb{B}}=0\right\} .
\end{aligned}
$$

We have embeddings $C_{r}^{1} \hookrightarrow W_{r}$ and $W_{r} \hookrightarrow C_{r}$, since $N \leq 3<p$. Here the second arrow is the usual Sobolev embedding restricted to radial functions. We also put

$$
X=W_{r} \times W_{r}, \quad Y=C_{r}^{1} \times C_{r}^{1}, \quad X_{+}=\{(u, v) \in X: u, v \geq 0\} .
$$

We remark that, if the pair $(u, v) \in X_{+}$is a weak solution of the coupled equations

$$
-\Delta u+\left(1-\beta v^{2}\right) u=u^{3} \geq 0, \quad-\Delta v+\left(1-\beta u^{2}\right) v=v^{3} \geq 0 \quad \text { in } \mathbb{B}
$$


and $u \neq 0, v \neq 0$, then $(u, v)$ is a solution of (1.3) by the strong maximum principle. We now collect some results on the parabolic problem

$$
\left\{\begin{array}{lr}
u_{t}-\Delta u+u-u^{3}-\beta v^{2} u=0 & \text { in } \mathbb{B}, \\
v_{t}-\Delta v+v-v^{3}-\beta u^{2} v=0 & \text { in } \mathbb{B}, \\
u=v=0 \quad \text { on } \partial \mathbb{B}, \quad u(0, x)=u_{0}(x), & v(0, x)=v_{0}(x) .
\end{array}\right.
$$

For the Cauchy problem (2.3) in the space $X$, we have the following.

Proposition 2.2. For every $\left(u_{0}, v_{0}\right) \in X$, the Cauchy problem (2.3) has a unique (mild) solution $(u(t), v(t))=\varphi\left(t, u_{0}, v_{0}\right) \in C([0, T), X)$ with maximal existence time $T:=T\left(u_{0}, v_{0}\right)>0$ which is a classical solution for $0<t<T$. The set $\mathcal{G}:=$ $\left\{\left(t, u_{0}, v_{0}\right): 0 \leq t<T\left(u_{0}, v_{0}\right)\right\}$ is open in $[0, \infty) \times X$, and $\varphi$ is a semiflow on $\mathcal{G}$.

Moreover we have:

(i) For every $\left(u_{0}, v_{0}\right) \in X$ and every $0<t<T\left(u_{0}, v_{0}\right)$ there is a neighborhood $U \subset X$ of $\left(u_{0}, v_{0}\right)$ in $X$ such that $T(u, v)>t$ for $(u, v) \in U$, and $\varphi(t, \cdot, \cdot):\left(U,\|\cdot\|_{X}\right) \rightarrow$ $\left(Y,\|\cdot\|_{Y}\right)$ is a continuous map.

(ii) If $\left(u_{0}, v_{0}\right) \in X_{+}$, then $\varphi\left(t, u_{0}, v_{0}\right) \in X_{+}$for $0 \leq t<T\left(u_{0}, v_{0}\right)$.

Proof. The proposition can be derived from abstract results of Amann concerning local existence and regularity, see [1]. For this we note that the substitution operator $F_{*}$ induced by the nonlinearity

$$
(u, v) \mapsto F(u, v)=\left(u-u^{3}-\beta v^{2} u, v-v^{3}-\beta u^{2} v\right) .
$$

is locally Lipschitz continuous as a map $W^{\tau, p}(\mathbb{B}) \times W^{\tau, p}(\mathbb{B}) \rightarrow L^{p}(\mathbb{B}) \times L^{p}(\mathbb{B})$ whenever $\tau>\frac{N}{p}$. Hence the local existence, the semiflow properties of $\varphi$ and (i) follow from $[1$, Theorem 2.1 and Theorem 2.4].

Property (ii) is just a consequence of the parabolic maximum principle, since $u$ and $v$ both satisfy equations of the form $w_{t}-\Delta w=f(x, t) w$ in $\mathbb{B}$ with locally bounded $f$, together with homogeneous Dirichlet boundary conditions.

In the following we will frequently write $\varphi^{t}(u)$ instead of $\varphi(t, u)$. For a classical solution of (2.3), we have

$$
\begin{aligned}
\frac{d}{d t} E(u, v) & =\int_{\mathbb{B}}\left(\nabla u \nabla u_{t}+\left(u-u^{3}-\beta v^{2} u\right) u_{t}\right) d x+\int_{\mathbb{B}}\left(\nabla v \nabla v_{t}+\left(v-v^{3}-\beta u^{2} v\right) v_{t}\right) d x \\
& =\int_{\mathbb{B}}\left(-\Delta u+u-u^{3}-\beta v^{2} u\right) u_{t} d x+\int_{\mathbb{B}}\left(-\Delta v+v-v^{3}-\beta u^{2} v\right) v_{t} d x \\
(2.5) \quad & =-\int_{\mathbb{B}}\left[\left(u_{t}\right)^{2}+\left(v_{t}\right)^{2}\right] d x,
\end{aligned}
$$

hence $E$ is strictly decreasing along non-constant trajectories $t \mapsto \varphi^{t}\left(u_{0}, v_{0}\right)$ in $X$. We need the following compactness property.

Proposition 2.3. Let $\left(u_{0}, v_{0}\right) \in X$ and $T=T\left(u_{0}, v_{0}\right)$ be such that the function $t \mapsto E\left(\varphi^{t}\left(u_{0}, v_{0}\right)\right)$ is bounded from below in $(0, T)$. Then $T=\infty$, and for every $\delta>0$ the set $\left\{\varphi^{t}\left(u_{0}, v_{0}\right): t \geq \delta\right\}$ is relatively compact in $Y$. 
Proof. Let $(u(t), v(t))=\varphi^{t}\left(u_{0}, v_{0}\right)$, and recall that the nonlinearity $F$ defined in $(2.4)$ has cubic growth. Hence, in view of Amann's abstract criterion for global existence and relative compactness (see [1, Theorem 5.3 and Remark 5.4]), it suffices to show that

$$
\sup _{0 \leq t<T}\left(|u(t)|_{\lambda}+|v(t)|_{\lambda}\right)<\infty \quad \text { for some } \lambda \text { satisfying } 3<1+\frac{2}{N} \lambda .
$$

We restrict our attention to the case $N=3$, since the case $N \leq 2$ is easier. We claim that (2.6) holds with $\lambda=\frac{10}{3}$. The following argument is similar to the method in [7], see in particular estimates (2.12) and (2.15) below. To shorten notation, we put $E_{\mathrm{inf}}=\inf _{0<t<T} E(u(t), v(t))$,

$$
\dot{E}=\frac{d}{d t} E(u, v)=-\left(\left|u_{t}\right|_{2}^{2}+\left|v_{t}\right|_{2}^{2}\right) \quad \text { and } \quad h=|u|_{2}^{2}+|v|_{2}^{2} .
$$

Then

$$
\frac{d h}{d t}=2 \int_{\mathbb{B}}\left(u u_{t}+v v_{t}\right) d x \leq 2\left(|u|_{2}\left|u_{t}\right|_{2}+|v|_{2}\left|v_{t}\right|_{2}\right) \leq h-\dot{E}
$$

and, by multiplying (2.3) with $u$ resp. $v$ and integrating,

$$
\begin{aligned}
\int_{\mathbb{B}}\left(u u_{t}+v v_{t}\right) d x & =-\left(\|u\|^{2}+\|v\|^{2}\right)+|u|_{4}^{4}+|v|_{4}^{4}+2 \beta|u v|_{2}^{2} \\
& =-4 E(u, v)+\|u\|^{2}+\|v\|^{2} .
\end{aligned}
$$

Consequently,

$$
\begin{aligned}
\|u\|^{2}+\|v\|^{2} & \leq 4 E\left(u_{0}, v_{0}\right)+\int_{\mathbb{B}}\left(u u_{t}+v v_{t}\right) d x \leq C_{1}+|u|_{2}\left|u_{t}\right|_{2}+|v|_{2}\left|v_{t}\right|_{2} \\
& \leq C_{1}+\sqrt{h}\left(\left|u_{t}\right|_{2}+\left|v_{t}\right|_{2}\right) .
\end{aligned}
$$

Here and in the following, $C_{1}, C_{2}, \ldots$ are positive constants independent of $t$. We first consider the case where $T<\infty$. From (2.7) we derive

so that

$$
\frac{d}{d t}\left(e^{-t} h(t)\right)=e^{-t}\left(\frac{d h}{d t}(t)-h(t)\right) \leq-e^{-t} \dot{E}(t) \leq-\dot{E}(t),
$$

$$
h(t) \leq e^{t}\left(h(0)-\int_{0}^{t} \dot{E}(s) d s\right) \leq e^{T}\left[h(0)+E\left(u_{0}, v_{0}\right)-E_{\text {inf }}\right] \leq C_{2}
$$

for $t \in[0, T)$. Hence (2.9) implies

$$
\|u(t)\|^{2}+\|v(t)\|^{2} \leq C_{3}\left(1+\left|u_{t}(t)\right|_{2}+\left|v_{t}(t)\right|_{2}\right)
$$

and therefore

(2.10) $\|u(t)\|^{4}+\|v(t)\|^{4} \leq C_{4}\left(1+\left|u_{t}(t)\right|_{2}^{2}+\left|v_{t}(t)\right|_{2}^{2}\right)=C_{4}(1-\dot{E}(t)) \quad$ for $t \in[0, T)$.

Thus we obtain for $0 \leq t<T$

$$
\int_{0}^{t}\left(\|u\|^{4}+\|v\|^{4}\right) d s \leq C_{4}\left[T+E\left(u_{0}, v_{0}\right)-E_{\text {inf }}\right]=: C_{5}
$$


which implies, for $\lambda=\frac{10}{3}$,

$$
\begin{aligned}
\frac{1}{\lambda}\left(|u(t)|_{\lambda}^{\lambda}\right. & \left.+|v(t)|_{\lambda}^{\lambda}-\left(|u(0)|_{\lambda}^{\lambda}+|v(0)|_{\lambda}^{\lambda}\right)\right)=\int_{0}^{t}\left(|u|^{\lambda-2} u u_{t}+|v|^{\lambda-2} v v_{t}\right) d s \\
& \leq \int_{0}^{t}\left(|u|_{2}^{\frac{1}{3}}|u|_{6}^{2}\left|u_{t}\right|_{2}+|v|_{2}^{\frac{1}{3}}|v|_{6}^{2}\left|v_{t}\right|_{2}\right) d s \leq \int_{0}^{t} h^{\frac{1}{6}}\left(|u|_{6}^{2}\left|u_{t}\right|_{2}+|v|_{6}^{2}\left|v_{t}\right|_{2}\right) d s \\
& \leq C_{6} \int_{0}^{t}\left(|u|_{6}^{4}+\left|u_{t}\right|_{2}^{2}+|v|_{6}^{4}+\left|v_{t}\right|_{2}^{2}\right) d s \leq C_{7} \int_{0}^{t}\left(\|u\|^{4}+\|v\|^{4}-\dot{E}\right) d s \\
& \leq C_{7}\left[C_{5}+E\left(u_{0}, v_{0}\right)-E_{\mathrm{inf}}\right]=: C_{8} .
\end{aligned}
$$

Here we used the Sobolev embedding $H_{r} \hookrightarrow L^{6}(\mathbb{B})$. This concludes the proof of $(2.6)$ if $T<\infty$.

Next we consider the case $T=\infty$. Then there exists a sequence $\left(t_{n}\right)_{n}$ with $n \leq t_{n} \leq$ $n+1$ and

$$
-\left(\left|u_{t}\left(t_{n}\right)\right|_{2}^{2}+\left|v_{t}\left(t_{n}\right)\right|_{2}^{2}\right)=\dot{E}\left(t_{n}\right) \rightarrow 0 \quad \text { as } n \rightarrow \infty .
$$

Combining this with (2.9), we get

$\left\|u\left(t_{n}\right)\right\|^{2}+\left\|v\left(t_{n}\right)\right\|^{2} \leq C_{1}+\sqrt{h\left(t_{n}\right)}\left(\left|u_{t}\left(t_{n}\right)\right|_{2}+\left|v_{t}\left(t_{n}\right)\right|_{2}\right) \leq C_{1}+o(1) \sqrt{\left\|u\left(t_{n}\right)\right\|^{2}+\left\|v\left(t_{n}\right)\right\|^{2}}$

which implies that

$$
\left\|u\left(t_{n}\right)\right\|+\left\|v\left(t_{n}\right)\right\| \leq C_{9} \quad \text { for all } n .
$$

Moreover, for $t_{n} \leq t \leq t_{n+1}$, we derive from (2.7)

$$
\frac{\partial}{\partial t}\left(e^{-\left(t-t_{n}\right)} h(t)\right)=e^{-\left(t-t_{n}\right)}\left(\frac{\partial h}{\partial t}(t)-h(t)\right) \leq-e^{-\left(t-t_{n}\right)} \dot{E}(t) \leq-\dot{E}(t),
$$

so that, by (2.13),

$$
h(t) \leq e^{t-t_{n}}\left(h\left(t_{n}\right)-\int_{t_{n}}^{t} \dot{E}(s) d s\right) \leq e^{2}\left(C_{9}^{2}+E\left(u_{0}, v_{0}\right)-E_{\text {inf }}\right) \leq C_{10} .
$$

Hence (2.9) implies

$$
\|u(t)\|^{2}+\|v(t)\|^{2} \leq C_{11}\left(1+\left|u_{t}(t)\right|_{2}+\left|v_{t}(t)\right|_{2}\right)
$$

and therefore

$$
\|u(t)\|^{4}+\|v(t)\|^{4} \leq C_{12}(1-\dot{E}(t))
$$

for all $t \geq 0$. Thus we obtain, for $t_{n} \leq t \leq t_{n+1}$, as in (2.11),

$$
\int_{t_{n}}^{t}\left(\|u\|^{4}+\|v\|^{4}\right) d s \leq C_{13}
$$


and thus, similarly as before,

$$
\begin{aligned}
\frac{1}{\lambda}\left(|u(t)|_{\lambda}^{\lambda}+|v(t)|_{\lambda}^{\lambda}\right) & =\frac{1}{\lambda}\left(\left|u\left(t_{n}\right)\right|_{\lambda}^{\lambda}+\left|v\left(t_{n}\right)\right|_{\lambda}^{\lambda}\right)+\int_{t_{n}}^{t}\left(|u|^{\lambda-2} u u_{t}+|v|^{\lambda-2} v v_{t}\right) d s \\
& \leq \frac{1}{\lambda}\left(\left|u\left(t_{n}\right)\right|_{\lambda}^{\lambda}+\left|v\left(t_{n}\right)\right|_{\lambda}^{\lambda}\right)+C_{14} \int_{t_{n}}^{t}\left(\|u\|^{4}+\|v\|^{4}-\dot{E}\right) d s \\
& \leq \frac{1}{\lambda}\left(\left|u\left(t_{n}\right)\right|_{\lambda}^{\lambda}+\left|v\left(t_{n}\right)\right|_{\lambda}^{\lambda}\right)+C_{15} \leq C_{16},
\end{aligned}
$$

where we used (2.13) and the Sobolev embedding $H_{r} \hookrightarrow L^{\lambda}(\mathbb{B})$ in the last step. The proof of (2.6) finished, and hence the claim follows.

The following Corollary is a consequence of (2.5) and Proposition 2.3.

Corollary 2.4. If, for some $\left(u_{0}, v_{0}\right) \in X_{+}$and $T=T\left(u_{0}, v_{0}\right)$, the function $t \mapsto$ $E\left(\varphi^{t}\left(u_{0}, v_{0}\right)\right)$ is bounded from below on $(0, T)$, then $T=\infty$ and the $\omega$-limit set

$$
\omega\left(u_{0}, v_{0}\right)=\bigcap_{t>0} \operatorname{clos} Y\left(\left\{\varphi^{s}\left(u_{0}, v_{0}\right): s \geq t\right\}\right)
$$

is a nonempty compact subset of $Y$ consisting of radial solutions of (1.3). Here $\operatorname{clos}_{Y}$ stands for the closure with respect to the $Y$-topology.

We also need a variant of Sturm's lap number theorem similar to the one available for scalar parabolic equations, see $[4,18,26,31]$ for the one-dimensional case and [32] for the radial case in higher dimensions. Given $(u, v) \in X$, we define the number of (strict) intersections $i(u, v)$ of $u$ and $v$ as the maximal $k \in \mathbb{N} \cup\{0, \infty\}$ such that there exist points $x_{1}, \ldots, x_{k+1} \in \mathbb{B}$ with $0 \leq\left|x_{1}\right|<\cdots<\left|x_{k+1}\right|<1$ and

$$
\left[u\left(x_{i}\right)-v\left(x_{i}\right)\right]\left[u\left(x_{i+1}\right)-v\left(x_{i+1}\right)\right]<0 \quad \text { for } i=1, \ldots, k .
$$

Lemma 2.5. Let $\left(u_{0}, v_{0}\right) \in X$ and $T:=T\left(u_{0}, v_{0}\right)$. Then $t \mapsto i\left(\varphi^{t}\left(u_{0}, v_{0}\right)\right)$ is nonincreasing in $t \in[0, T)$.

This Lemma can easily be derived from [32, Theorem 2.1]. In fact, the general result in [32] for scalar equations implies a stronger monotonicity property than the one stated in Lemma 2.5. Since we only need the weak version stated above, we give a short proof following an argument of Sattinger (cf. [34, Theorem 4]).

Proof. We write $(u(t), v(t))=\varphi^{t}\left(u_{0}, v_{0}\right)$, so that $(u, v)$ is a solution of (2.3). In view of the semiflow properties, it suffices to show the inequality $i(u(\tau), v(\tau)) \leq i\left(u_{0}, v_{0}\right)$ for fixed $0<\tau<T$. We consider the function $\tilde{w}=u-v$ which is continuous on $\mathbb{B} \times[0, \tau]$ and satisfies the equation $\tilde{w}_{t}-\Delta \tilde{w}+f(x, t) \tilde{w}=0$ in $\mathbb{B} \times(0, \tau]$, where $f(\cdot, t)=1-\left[u^{2}(t)+v^{2}(t)\right]+(\beta-1) u(t) v(t)$ is bounded in $\mathbb{B} \times[0, \tau]$. Fix $\lambda>0$ such that $g(x, t):=f(x, t)+\lambda$ is positive on $\mathbb{B} \times[0, \tau]$, and consider $w(x, t)=e^{-\lambda t} \tilde{w}(x, t)$. Then $w$ is continuous on $\mathbb{B} \times[0, \tau]$ and satisfies the equation

$$
w_{t}-\Delta w+g(x, t) w=0 \quad \text { in } \mathbb{B} \times(0, \tau] .
$$

Let

$U^{+}=\{(x, t) \in \mathbb{B} \times[0, \tau]: w(x, t)>0\}, \quad U^{-}=\{(x, t) \in \mathbb{B} \times[0, \tau]: w(x, t)<0\}$. 
We show that every connected component of $U^{+}$intersects $S_{0}:=\mathbb{B} \times\{0\}$. Indeed, suppose by contradiction that there is a component $U$ such that $U \cap S_{0}=\varnothing$. Since $w \equiv 0$ on the relative boundary of $U$ in $\mathbb{B} \times[0, \tau]$, there exists $\left(x_{0}, t_{0}\right) \in U$ with $w\left(x_{0}, t_{0}\right)=\max _{U} w>0$. Hence $\Delta w\left(x_{0}, t_{0}\right) \leq 0$. Moreover, since $t_{0}>0$, we have $w_{t}\left(x_{0}, t_{0}\right)=0$ if $t_{0}<\tau$ and $w_{t}\left(x_{0}, t_{0}\right) \geq 0$ if $t_{0}=\tau$. This however contradicts (2.16), since $g>0$ on $\mathbb{B} \times[0, \tau]$. Similarly, we show that every connected component of $U^{-}$ intersects $S_{0}$.

Now let $k=i(u(\tau), v(\tau))$, and choose $x_{1}, \ldots, x_{k+1}$ with $0 \leq\left|x_{1}\right|<\cdots<\left|x_{k+1}\right|<1$ and

$$
w\left(x_{i}, \tau\right) w\left(x_{i+1}, \tau\right)<0 \quad \text { for } i=1, \ldots, k .
$$

We may assume that $w\left(x_{1}, \tau\right)>0$ and that $k+1=2 j$ is even, the other cases are treated similarly. Then there are corresponding components $U_{1}^{+}, \ldots, U_{j}^{+}$of $U^{+}$and $U_{1}^{-}, \ldots, U_{j}^{-}$of $U^{-}$such that $\left(x_{2 i-1}, \tau\right) \in U_{i}^{+}$and $\left(x_{2 i}, \tau\right) \in U_{i}^{-}$for $i=1, \ldots, j$. Since $U_{i}^{ \pm} \cap S_{0} \neq \varnothing$ for every $i$, we may pick $\left(y_{2 i-1}, 0\right) \in U_{i}^{+} \cap S_{0}$ and $\left(y_{2 i}, 0\right) \in U_{i}^{-} \cap S_{0}$. From the fact that $w(\cdot, t)$ is a radial function for all $0 \leq t \leq \tau$, we deduce that $0 \leq\left|y_{1}\right|<\left|y_{2}\right|<\cdots<\left|y_{k+1}\right|$, while $w\left(y_{i}, 0\right) w\left(y_{i+1}, 0\right)<0$ for $i=1, \ldots, k$. Hence $i\left(u_{0}, v_{0}\right) \geq k$, as claimed.

By Proposition 2.2 and the principle of linearized stability, the constant solution $(u, v) \equiv(0,0)$ is stable in $X$, so that the set

$$
\mathcal{A}_{*}:=\left\{(u, v) \in X_{+}: T(u, v)=\infty \text { and } \varphi^{t}(u, v) \rightarrow(0,0) \text { in } X \text { as } t \rightarrow \infty\right\}
$$

is a relatively open neighborhood of $(0,0)$ in $X_{+}$.

Lemma 2.6. $\left\{(u, u): u \in W_{r}, u \geq 0\right\} \subset \mathcal{A}_{*}$.

Proof. Let $u_{0} \in W_{r}, u_{0} \geq 0$. By uniqueness of the solution of the Cauchy problem (1.3), we have $\varphi^{t}\left(u_{0}, u_{0}\right)=(u(x, t), u(x, t))$, where $u(x, t)$ is the unique solution of the Cauchy problem

$$
u_{t}-\Delta u=(1+\beta) u^{3}-u \quad \text { in } \mathbb{B}, \quad u=0 \quad \text { on } \partial \mathbb{B}, \quad u(0)=u_{0} .
$$

A comparison with the solution $y=y(t)$ of the ordinary differential equation $\dot{y}=$ $(1+\beta) y^{3}-y$ satisfying $y(0)=\left|u_{0}\right|_{\infty}$ yields $0 \leq u(x, t) \leq y(t)$ for all $x \in \mathbb{B}, t \geq 0$, whereas $y(t) \rightarrow 0$ as $t \rightarrow \infty$ since $\beta \leq-1$. This shows that $|u(\cdot, t)|_{\infty}$ is uniformly bounded in $t \in\left[0, T\left(u_{0}, u_{0}\right)\right)$, so that $E\left(\varphi^{t}\left(u_{0}, u_{0}\right)\right)$ remains bounded from below. Hence $T\left(u_{0}, u_{0}\right)=\infty$ by Proposition 2.3 , and for $\delta>0$ the set $\left\{\varphi^{t}\left(u_{0}, u_{0}\right): t \geq \delta\right\}$ is relatively compact in $Y$. Since $|u(\cdot, t)|_{\infty} \leq y(t) \rightarrow 0$ as $t \rightarrow \infty$, we conclude that $\varphi^{t}\left(u_{0}, u_{0}\right) \rightarrow 0$ in the $Y$-topology and therefore also in the $X$-topology. Hence $\left(u_{0}, u_{0}\right) \in \mathcal{A}_{*}$, as claimed.

\section{Existence OF SOLUTIONS With A GIVEN NUMBER OF INTERSECTIONS}

We keep using the notation of Section 2. Let $\partial \mathcal{A}_{*}$ denote the relative boundary of the set $\mathcal{A}_{*}$ (see (2.17)) in $X_{+}$with respect to the $X$-topology. The continuity of the semiflow $\varphi$ and Proposition 2.2(ii) imply that $\partial \mathcal{A}_{*}$ is positively invariant under 
$\varphi$. Moreover, $E(u, v) \geq 0$ and $T(u, v)=\infty$ for every $(u, v) \in \partial \mathcal{A}_{*}$ by Proposition 2.3. We now define

$Y_{k}:=\{(u, v) \in Y: i(u, v) \leq k-1\} \quad$ and $\quad \mathcal{A}_{k}:=\left\{(u, v) \in \partial \mathcal{A}_{*}: i(u, v) \leq k-1\right\}$

By definition, $\mathcal{A}_{k}$ is a closed subset of $X$, and by Lemma 2.5 it is a positively invariant set for the flow $\varphi$. Our aim is to find solutions of (1.3) in $\mathcal{A}_{k} \backslash \mathcal{A}_{k-1}$ for every $k \geq 2$.

We remark the following.

Lemma 3.1. If $(u, v) \in \mathcal{A}_{k}$ is a radial solution of (1.3), then $(u, v) \in \operatorname{int}_{Y}\left(Y_{k}\right)$, where $\operatorname{int}_{Y}\left(Y_{k}\right)$ denotes the interior of $Y_{k}$ with respect to the $Y$-topology.

Proof. If $(u, v)$ is a radial solution of (1.3), then $(u, v) \in Y$ by standard elliptic regularity. Moreover, as a function of the radial variable, $w=u-v$ is a solution of the one-dimensional boundary value problem

$$
-w_{r r}-\frac{N-1}{r} w_{r}+f(r) w=0, \quad r \in(0,1), \quad w_{r}(0)=0, w(1)=0,
$$

where $f(r)=1-\left[u^{2}(r)+v^{2}(r)\right]+(\beta-1) u(r) v(r)$. Hence $w(0) \neq 0$, and $r \mapsto w(r)$ has only simple zeros in $(0,1]$. In fact, $w$ has $l \leq k-1$ zeros since $(u, v) \in \mathcal{A}_{k}$. But then there is a neighborhood of $w$ in the $C^{1}$-topology containing only functions with precisely $l$ simple zeros. Hence $(u, v) \in \operatorname{int}_{Y}\left(Y_{k}\right)$, as claimed.

Next we note that the set $\partial \mathcal{A}_{*}$ and the sets $\mathcal{A}_{k}, k \geq 1$ are symmetric with respect to the involution $(u, v) \mapsto \sigma(u, v)=(v, u)$, and the semiflow $\varphi^{t}$ is $\sigma$-equivariant. We also note that $\sigma$ has no fixed points in $\partial \mathcal{A}_{*}$ by Lemma 2.6. For a closed $\sigma$-symmetric subset $A \subset \partial \mathcal{A}_{*}$ we define the genus $\gamma(A)$ corresponding to $\sigma$ as the least $k \in \mathbb{N} \cup\{0\}$ such that there is a continuous map $h: A \rightarrow \mathbb{R}^{k} \backslash\{0\}$ with $h(v, u)=-h(u, v)$. As usual, we define $\gamma(A)=\infty$ if no such $k$ exists. The genus has many useful properties. In the following we only list the properties we need.

Lemma 3.2. Let $A, B \subset \partial \mathcal{A}_{*}$ be closed and $\sigma$-symmetric.

(i) If $A \subset B$, then $\gamma(A) \leq \gamma(B)$.

(ii) $\gamma(A \cup B) \leq \gamma(A)+\gamma(B)$.

(iii) If $h: A \rightarrow \partial \mathcal{A}_{*}$ is continuous and $\sigma$-equivariant, then $\gamma(A) \leq \gamma(\overline{h(A)})$.

(iv) If $\gamma(A)<\infty$, then there exists a relatively open $\sigma$-symmetric neighborhood $N$ of $A$ in $\partial \mathcal{A}_{*}$ such that $\gamma(A)=\gamma(\bar{N})$.

(v) If $S$ is the boundary of a bounded symmetric neighborhood of the origin in a $k$-dimensional normed vector space und $\psi: S \rightarrow \partial \mathcal{A}_{*}$ is a continuous map satisfying $\psi(-u)=\sigma(\psi(u))$, then $\gamma(\psi(S)) \geq k$.

Note that in (v) the set $\psi(S)$ is closed since $S$ is compact.

Proof. Properties (i) and (iii) follow immediately from the definition of $\gamma$. Moreover, (ii) and (iv) can be proved using the Tietze extension theorem similarly as in [42, p. 96]. Property (v) is proved by contradiction, assuming that there exists a continuous map $h: \psi(S) \rightarrow \mathbb{R}^{k-1} \backslash\{0\}$ with $h(v, u)=-h(u, v)$. Then $h \circ \psi: S \rightarrow \mathbb{R}^{k-1} \backslash\{0\}$ is an odd and continuous map, which contradicts the Borsuk-Ulam Theorem (see e.g. [44, Theorem D.17.]). 
Lemma 3.3. $\gamma\left(\mathcal{A}_{k}\right) \leq k$.

Proof. We proceed by induction, starting with $k=1$. By definition, $\mathcal{A}_{1}$ is precisely the set of vectors $(u, v) \in \partial \mathcal{A}_{*}$ such that $u-v$ does not change sign. By Lemma 2.6, $\left\{(u, u): u \in W_{r}, u \geq 0\right\} \cap \mathcal{A}_{1}=\varnothing$, which implies that $\mathcal{A}_{1}=B_{+} \cup B_{-}$with disjoint subsets $B_{ \pm}$defined by

$B_{+}=\left\{(u, v) \in \mathcal{A}_{*}: u \geq v, u-v \not \equiv 0\right\}, \quad B_{-}=\left\{(u, v) \in \mathcal{A}_{*}: u \leq v, u-v \not \equiv 0\right\}$.

Since the sets $\mathcal{B}_{ \pm}$are relatively open in $\mathcal{A}_{1}$, the map

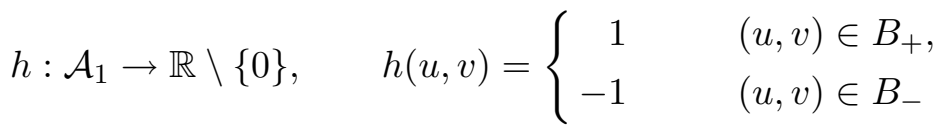

is continuous, and it is also $\sigma$-symmetric. We conclude that $\gamma\left(\mathcal{A}_{1}\right) \leq 1$, as claimed. Next we consider $k>1$ and assume that $\gamma\left(\mathcal{A}_{k-1}\right) \leq k-1$. We use the fact that $\mathcal{A}_{k}=\tilde{A} \cup \mathcal{A}_{k-1}$, where $\tilde{A}=\left\{(u, v) \in \mathcal{A}_{*}: i(u, v)=k-1\right\}$. Let $\tilde{B}_{ \pm}$be the set of all $(u, v) \in \tilde{A}$ such that, for some $x_{1} \in \mathbb{B}$,

$$
\pm\left(u\left(x_{1}\right)-v\left(x_{1}\right)\right)>0 \quad \text { and } \quad \pm(u(x)-v(x)) \geq 0 \quad \text { for } 0 \leq x \leq\left|x_{1}\right| .
$$

Then $\tilde{A}=\tilde{B}_{+} \cup \tilde{\mathcal{B}}_{-}$. We claim that the sets $\tilde{B}_{ \pm}$are relatively open in $\tilde{A}$. Indeed, if $(u, v) \in \tilde{B}_{+}$, then there are points $x_{1}, \ldots, x_{k}$ with $0 \leq\left|x_{1}\right|<\cdots<\left|x_{k}\right|<1$ such that

$$
u(x)-v(x) \geq 0, \quad u\left(x_{1}\right)-v\left(x_{1}\right)>0, \quad \text { and }\left[u\left(x_{i}\right)-v\left(x_{i}\right)\right]\left[u\left(x_{i+1}\right)-v\left(x_{i+1}\right)\right]<0
$$

for $0 \leq|x| \leq\left|x_{1}\right|$ and $i=1, \ldots, k-1$. Hence there is a neighborhood $U \subset X_{+}$of $(u, v)$ such that $\left[\tilde{u}\left(x_{i}\right)-\tilde{v}\left(x_{i}\right)\right]\left[\tilde{u}\left(x_{i+1}\right)-\tilde{v}\left(x_{i+1}\right)\right]<0$ for every $(\tilde{u}, \tilde{v}) \in U, i=1, \ldots, k-1$. This implies that $\tilde{u}(x)-\tilde{v}(x) \geq 0$ for $0 \leq|x| \leq\left|x_{1}\right|$ and every $(\tilde{u}, \tilde{v}) \in U \cap \tilde{B}_{+}$, since $i(\tilde{u}, \tilde{v})=k-1$. Hence $\tilde{B}_{+}$is relatively open in $\tilde{A}$. A similar argument shows that $\tilde{B}_{-}$ is relatively open in $\tilde{A}$. Consequently, the map

$$
\tilde{h}: \tilde{A} \rightarrow \mathbb{R} \backslash\{0\}, \quad \tilde{h}(u, v)=\left\{\begin{aligned}
1 & (u, v) \\
-1 & (u, v) \in \tilde{B}_{+}, \\
-1 & \tilde{B}_{-}
\end{aligned}\right.
$$

is continuous and $\sigma$-symmetric. To conclude the proof, we let $N \subset \partial \mathcal{A}_{*}$ be a relatively open $\sigma$-symmetric neighborhood of $\mathcal{A}_{k-1}$ such that

$$
\gamma(\bar{N})=\gamma\left(\mathcal{A}_{k-1}\right) \leq k-1,
$$

as provided by Lemma 3.2(iv). Since $\mathcal{A}_{k} \backslash N$ is a closed $\sigma$-symmetric subset of $\tilde{A}$ and therefore $\gamma\left(\mathcal{A}_{k} \backslash N\right) \leq 1$ via the map $\tilde{h}$ defined above, we conclude that

$$
\gamma\left(\mathcal{A}_{k}\right) \leq \gamma(\bar{N})+\gamma\left(\mathcal{A}_{k} \backslash N\right) \leq k .
$$

Proposition 3.4. For every $k \geq 2$, there exists a solution $(u, v) \in \mathcal{A}_{k} \backslash \mathcal{A}_{k-1}$ of (1.3) with $E(u, v) \leq c_{k}$. 
Proof. It is known (see $[40,41])$ that there is a radial solution $\bar{w}$ of the equation

$$
\Delta w-w+w^{3}=0 \quad \text { in } \mathbb{B}, \quad w=0 \quad \text { on } \partial \mathbb{B}
$$

with $E_{S}(\bar{w})=c_{k}$ and such that $\bar{w}$, viewed as a function of the radial variable, has precisely $k-1$ interior zeros $0<r_{1}<\cdots<r_{k-1}<1$. Put $r_{0}=0$ and $r_{k}=1$, and consider $w_{j}=\bar{w} \cdot 1_{\left\{r_{j} \leq|x| \leq r_{j+1}\right\}} \in W_{r}$ for $j=0, \ldots, k-1$. Multiplying (3.1) by $w_{j}$ and integrating over $\left\{r_{j} \leq|x| \leq r_{j+1}\right\}$, we find that $\left\|w_{j}\right\|^{2}=\left|w_{j}\right|_{4}^{4}$ and therefore $E_{S}\left(w_{j}\right)=\frac{1}{4}\left\|w_{j}\right\|^{2}$. Hence we have

$$
E_{S}\left(s w_{j}\right)=\frac{1}{2}\left(s^{2}-\frac{s^{4}}{2}\right)\left\|w_{j}\right\|^{2} \leq \frac{1}{4}\left\|w_{j}\right\|^{2}=E_{S}\left(w_{j}\right) \quad \text { for every } s \in \mathbb{R}
$$

and

$$
E_{S}\left(s w_{j}\right) \rightarrow-\infty \quad \text { as }|s| \rightarrow \infty .
$$

We consider the $k$-dimensional subspace $W \subset W_{r}$ spanned by the functions $w_{j}, j=$ $0, \ldots, k-1$, and the map

$$
\psi: W \rightarrow X_{+}, \quad \psi(w)=\left(w^{+}, w^{-}\right),
$$

where $w^{+}=\max \{w, 0\}, w^{-}=-\min \{w, 0\}$. Clearly $\psi$ is continuous, and $\psi(-w)=$ $\sigma(\psi(w))$ for all $w \in W$. Using (3.2), we find that

$$
E\left(\psi\left(\sum_{j=1}^{k} s_{j} w_{j}\right)\right)=\sum_{j=1}^{k} E_{S}\left(s_{j} w_{j}\right) \leq \sum_{j=1}^{k} E_{S}\left(w_{j}\right)=E_{S}(\bar{w})=c_{k}
$$

for all $\left(s_{1}, \ldots, s_{k}\right) \in \mathbb{R}^{k}$, while

$$
\lim _{\|w\| \rightarrow \infty} E(\psi(w))=-\infty
$$

by (3.3). Hence $\mathcal{O}:=\left\{w \in W: \psi(w) \in \mathcal{A}_{*}\right\}$ is a symmetric bounded open neighborhood of 0 in $W$, and $\psi(\partial \mathcal{O}) \subset \mathcal{A}_{k}$. Lemma 3.2(v) implies that $\gamma(\psi(\partial \mathcal{O})) \geq k$. On the other hand, defining the closed subsets

$$
\mathcal{C}_{k-1}^{t}:=\left\{(u, v) \in \partial \mathcal{A}_{*}: \varphi^{t}(u, v) \in \mathcal{A}_{k-1}\right\} \subset \partial \mathcal{A}_{*} \quad \text { for } t>0,
$$

we infer $\gamma\left(\mathcal{C}_{k-1}^{t}\right) \leq k-1$ by Lemma 3.2 (iii) and Lemma 3.3 for every $t>0$. In particular, for every positive integer $n$ there exists $\left(u_{n}, v_{n}\right) \in \psi(\partial \mathcal{O}) \backslash \mathcal{C}_{k-1}^{n}$, so that $\varphi^{n}\left(u_{n}, v_{n}\right) \notin \mathcal{A}_{k-1}$. Since $\psi(\partial \mathcal{O})$ is compact, we may pass to a subsequence such that $\left(u_{n}, v_{n}\right) \rightarrow(\bar{u}, \bar{v})$ as $n \rightarrow \infty$. We claim that

$$
\varphi^{t}(\bar{u}, \bar{v}) \notin \operatorname{int}_{Y}\left(Y_{k-1}\right) \quad \text { for every } t>0 .
$$

Indeed, assuming by contradiction that $\varphi^{t_{0}}(\bar{u}, \bar{v}) \in \operatorname{int}_{Y}\left(Y_{k-1}\right)$ for some $t_{0}>0$, the continuity of $\varphi^{t}$ as stated in Proposition 2.2(i) implies that

$$
\varphi^{t_{0}}\left(u_{n}, v_{n}\right) \in \operatorname{int}_{Y}\left(Y_{k-1}\right) \cap \partial \mathcal{A}_{*} \subset \mathcal{A}_{k-1} \quad \text { for } n \text { large enough, }
$$

hence $\varphi^{n}\left(u_{n}, v_{n}\right) \in \mathcal{A}_{k-1}$ for $n$ large by the positive invariance of $\mathcal{A}_{k-1}$. This contradicts the choice of $\left(u_{n}, v_{n}\right)$. Hence (3.5) is true.

Now (3.5) implies that the $\omega$-limit set $\omega(\bar{u}, \bar{v})$ does not intersect $\operatorname{int}_{Y}\left(Y_{k-1}\right)$. Since $\omega(\bar{u}, \bar{v})$ consists of radial solutions of (1.3), we conclude by Lemma 3.1 that $\omega(\bar{u}, \bar{v}) \subset$ 
$\mathcal{A}_{k} \backslash \mathcal{A}_{k-1}$. Moreover, $E(u, v) \leq E(\bar{u}, \bar{v}) \leq c_{k}$ for every $(u, v) \in \omega(\bar{u}, \bar{v})$ by (3.4). So every $(u, v) \in \omega(\bar{u}, \bar{v})$ has the asserted properties.

Theorem 1.1 follows directly from Proposition 3.4.

\section{Asymptotic behaviour as $\beta \rightarrow \infty$}

This section is devoted to the proof of Theorem 1.2. For fixed $k \geq 2$, let $\beta_{n} \leq-1$, $n \in \mathbb{N}$ be such that $\beta_{n} \rightarrow-\infty$ as $n \rightarrow \infty$, and let $\left(u_{n}, v_{n}\right) \in H_{r} \times H_{r}$ be solutions of (1.3) with $\beta=\beta_{n}$ such that $u_{n}-v_{n}$ changes sign precisely $k-1$ times in the radial variable and $E\left(u_{n}, v_{n}\right) \leq c_{k}$. In the following, $C_{0}, C_{1}, \ldots$ always stand for positive constants independent of $n$. By (2.1), the energy bound yields a uniform $H^{1}$-bound for the sequence $\left(u_{n}, v_{n}\right)_{n}$. Passing to a subsequence, we may therefore assume that

$$
u_{n} \rightarrow u, \quad v_{n} \rightarrow v \quad \text { weakly in } H_{r} .
$$

Since $\beta_{n}$ is negative and $u_{n}, v_{n}$ are bounded in $H^{1}(\mathbb{B})$, we deduce from standard elliptic subsolution estimates (e.g. Theorem 8.17 of [16]) that

$$
\left|u_{n}\right|_{\infty},\left|v_{n}\right|_{\infty} \leq C_{0} .
$$

We consider the radial functions

$$
H_{n}: \mathbb{B} \rightarrow \mathbb{R}, \quad H_{n}:=\left|u_{n}^{\prime}\right|^{2}+\left|v_{n}^{\prime}\right|^{2}-\left(u_{n}^{2}+v_{n}^{2}\right)+\frac{1}{2}\left(u_{n}^{4}+v_{n}^{4}\right)+\beta_{n} u_{n}^{2} v_{n}^{2},
$$

where the prime stands for the radial derivative $\frac{d}{d r}$. The following monotonocity property in $r=|x|$ is crucial:

$$
\begin{aligned}
H_{n}^{\prime}(r) & =2 u_{n}^{\prime}(r)\left[u_{n}^{\prime \prime}(r)-u_{n}(r)+u_{n}^{3}(r)+\beta_{n} v_{n}^{2}(r) u_{n}(r)\right] \\
& +2 v_{n}^{\prime}(r)\left[v_{n}^{\prime \prime}(r)-v_{n}(r)+v_{n}^{3}(r)+\beta_{n} u_{n}^{2}(r) v_{n}(r)\right] \\
& =-\frac{2(N-1)}{r}\left(\left[u_{n}^{\prime}(r)\right]^{2}+\left[v_{n}^{\prime}(r)\right]^{2}\right) \leq 0 \quad \text { for } r>0 .
\end{aligned}
$$

The second equality follows from (1.3). Since $\beta_{n}<0$ and $u_{n}^{\prime}(0)=v_{n}^{\prime}(0)=0$, we have

$$
H_{n}(0) \leq \frac{1}{2}\left(u_{n}^{4}(0)+v_{n}^{4}(0)\right) \leq C_{1}
$$

and therefore

$$
0<\left|u_{n}^{\prime}(1)\right|^{2}+\left|v_{n}^{\prime}(1)\right|^{2}=H_{n}(1) \leq H_{n}(0) \leq C_{1}
$$

We thus conclude that the functions $H_{n}$ are positive, nonincreasing and uniformly bounded in $[0,1]$. Integrating, we also get

$$
C_{1} \geq H_{n}(0)-H_{n}(1)=2(N-1) \int_{0}^{1} \frac{\left[u_{n}^{\prime}(r)\right]^{2}+\left[v_{n}^{\prime}(r)\right]^{2}}{r} d r .
$$

Viewing $u_{n}, v_{n}$ as functions of $r \in[0,1]$, we deduce

$$
\left\|u_{n}\right\|_{H^{1}([0,1])},\left\|v_{n}\right\|_{H^{1}([0,1])} \leq C_{2}
$$

for $N \geq 2$, while for $N=1$ this is already known. We therefore conclude that

$$
u_{n} \rightarrow u, \quad v_{n} \rightarrow v \quad \text { uniformly in } \mathbb{B} .
$$


In particular, $u$ and $v$ are continuous. In the next three lemmas, we collect further properties of the sequence $\left(u_{n}, v_{n}\right)_{n}$ and its limit $(u, v)$.

Lemma 4.1. Let $P(u)=\{x \in \mathbb{B}: u(x)>0\}, P(v)=\{x \in \mathbb{B}: v(x)>0\}$.

(i) For any $\tau>0$,

$$
\begin{array}{ll}
\left|\beta_{n}\right|^{\tau} v_{n} \rightarrow 0 & \text { uniformly on compact subsets of } P(u), \\
\left|\beta_{n}\right|^{\tau} u_{n} \rightarrow 0 & \text { uniformly on compact subsets of } P(v) .
\end{array}
$$

(ii) On $P(u)$ resp. $P(v), u$ resp. $v$ solve the equations

$$
-\Delta u+u=u^{3}, \quad-\Delta v+v=v^{3},
$$

respectively, in classical sense.

The following proof does not use the radial symmetry of $u_{n}$ and $v_{n}$. It only relies on (4.6).

Proof. (i) We only prove the first statement. Let $K \subset P(u)$ be compact, and let $\varepsilon>0$ be such that

$$
K_{\varepsilon}:=\left\{x \in \mathbb{R}^{N}: \operatorname{dist}(x, K) \leq \varepsilon\right\} \subset\{x \in P(u): u(x)>\varepsilon\} .
$$

In $K_{\varepsilon}$, we have

$\Delta v_{n} \geq\left(1-v_{n}^{2}-\frac{\beta_{n} \varepsilon^{2}}{2}\right) v_{n} \geq\left(\frac{\left|\beta_{n}\right| \varepsilon^{2}}{2}-C_{3}\right) v_{n} \geq \frac{\left|\beta_{n}\right| \varepsilon^{2}}{4} v_{n} \quad$ for $n$ sufficiently large.

Now fix $x_{0} \in K$. Since $B_{\varepsilon}\left(x_{0}\right) \subset K_{\varepsilon}$, we have

$$
\left\{\begin{aligned}
\Delta v_{n} \geq M_{n} v_{n} & \text { in } B_{\varepsilon}\left(x_{0}\right), \\
v_{n} \geq 0 & \text { in } B_{\varepsilon}\left(x_{0}\right), \\
v_{n} \leq C_{0} & \text { on } \partial B_{\varepsilon}\left(x_{0}\right),
\end{aligned}\right.
$$

where $M_{n}:=\frac{\left|\beta_{n}\right| \varepsilon^{2}}{4}$. Applying [13, Lemma 4.4] with $\alpha=\frac{1}{2}$, we conclude that

$$
v_{n}\left(x_{0}\right) \leq C_{4} e^{-\frac{\varepsilon}{2} \sqrt{M_{n}}}=C_{4} e^{-\frac{\varepsilon^{2}}{4} \sqrt{\left|\beta_{n}\right|}} .
$$

For $n$ large enough such that $\sqrt{\left|\beta_{n}\right|} \geq \frac{8 \tau}{\varepsilon^{2}} \log \left|\beta_{n}\right|$, we conclude

$$
v_{n}\left(x_{0}\right) \leq C_{4}\left|\beta_{n}\right|^{-2 \tau}
$$

where the constant $C_{4}$ does not depend on $x_{0}$. Hence $\sup _{K}\left|\beta_{n}\right|^{\tau} v \rightarrow 0$ as $n \rightarrow \infty$, as claimed.

(ii) For $\varphi \in C_{0}^{\infty}(P(u))$ we have

$$
\begin{aligned}
\int_{P(u)} u \Delta \varphi d x & =\lim _{n \rightarrow \infty} \int_{P(u)} u_{n} \Delta \varphi d x=\lim _{n \rightarrow \infty} \int_{P(u)} \Delta u_{n} \varphi d x \\
& =\lim _{n \rightarrow \infty} \int_{P(u)}\left(u_{n}-u_{n}^{3}-\beta_{n} v_{n}^{2} u_{n}\right) \varphi d x=\int_{P(u)}\left(u-u^{3}\right) \varphi d x
\end{aligned}
$$

as a consequence of (i) and (4.6). Hence $u$ is a distributional solution of $-\Delta u+u=u^{3}$ in $P(u)$. Since we already know that $u$ is continuous, classical elliptic regularity shows that $u$ is in fact a classical solution. The statement for $v$ is proved in the same way. 


\section{Corollary 4.2.}

(i) If $0<r_{1}<r_{2} \leq 1$ are such that $u$ is positive in $\mathcal{A}:=\left\{x \in \mathbb{B}: r_{1}<|x|<r_{2}\right\}$ and $\left.u\right|_{\partial \mathcal{A}}=0$, then

$$
\int_{\mathcal{A}}\left(|\nabla u|^{2}+u^{2}-u^{4}\right) d x=0
$$

(ii) If $0<r \leq 1$ is such that $u$ is positive in $\mathcal{B}:=\{x \in \mathbb{B}:|x|<r\}$ and $\left.u\right|_{\partial \mathcal{B}}=0$, then

$$
\int_{\mathcal{B}}\left(|\nabla u|^{2}+u^{2}-u^{4}\right) d x=0
$$

Remark 4.3. The same statements are true for $v$ in place of $u$.

Proof. (i) Since $u$ is differentiable in $\mathcal{A} \subset \mathcal{P}(u)$ by Lemma 4.1(ii), we may pick $r_{1}<s_{n}<t_{n}<r_{2}$ such that $s_{n} \rightarrow r_{1}, t_{n} \rightarrow r_{2}$ as $n \rightarrow \infty$ and $u^{\prime}\left(s_{n}\right) \geq 0, u^{\prime}\left(t_{n}\right) \leq 0$ for all $n$. Then $\varepsilon_{n}:=\max \left\{u\left(s_{n}\right), u\left(t_{n}\right)\right\} \rightarrow 0$ as $n \rightarrow \infty$. Now Lemma 4.1(ii) implies that

$$
\begin{gathered}
\left|\int_{s_{n}<|x|<t_{n}}\left(|\nabla u|^{2}+u^{2}-u^{4}\right) d x\right|=\left|\int_{|x|=t_{n}} u \frac{\partial u}{\partial r} d \sigma-\int_{|x|=s_{n}} u \frac{\partial u}{\partial r} d \sigma\right| \\
\leq \varepsilon_{n}\left|\int_{|x|=t_{n}} \frac{\partial u}{\partial r} d \sigma-\int_{|x|=s_{n}} \frac{\partial u}{\partial r} d \sigma\right|=\varepsilon_{n}\left|\int_{s_{n}<|x|<t_{n}} \Delta u d x\right| \\
\leq \varepsilon_{n} \int_{\mathcal{A}}\left|u-u^{3}\right| d x \rightarrow 0 \quad \text { as } n \rightarrow \infty .
\end{gathered}
$$

Hence (4.7) follows. The proof of (ii) is similar.

\section{Lemma 4.4.}

(i) $u_{n} v_{n} \rightarrow u v=0$ uniformly in $\mathbb{B}$.

(ii) $\beta_{n} \int_{\mathbb{B}} u_{n}^{2} v_{n}^{2} d x \rightarrow 0$ as $n \rightarrow \infty$.

(iii) $\max \{u(0), v(0)\} \geq \sqrt{2}$.

Proof. (i) follows immediately from (4.6) and Lemma 4.1(i).

(ii) Since

$0 \leq-\int_{\partial \mathbb{B}} \frac{\partial u_{n}}{\partial r} d \sigma=-\int_{\mathbb{B}} \Delta u_{n} d x=\int_{\mathbb{B}}\left(u_{n}^{3}-u_{n}+\beta_{n} v_{n}^{2} u_{n}\right) d x \leq C_{5}-\left|\beta_{n}\right| \int_{\mathbb{B}} v_{n}^{2} u_{n} d x$, we have $\left|\beta_{n}\right| \int_{\mathbb{B}} v_{n}^{2} u_{n} d x \leq C_{5}$ and similarly $\left|\beta_{n}\right| \int_{\mathbb{B}} u_{n}^{2} v_{n} d x \leq C_{5}$. From (i) we therefore deduce

$\left|\beta_{n}\right| \int_{\mathbb{B}} u_{n}^{2} v_{n}^{2} d x \leq\left|\beta_{n}\right| \sqrt{\left|u_{n} v_{n}\right|_{\infty}} \int_{\mathbb{B}} u_{n} v_{n}\left(u_{n}+v_{n}\right) d x \leq 2 C_{5} \sqrt{\left|u_{n} v_{n}\right|_{\infty}} \rightarrow 0 \quad$ as $n \rightarrow \infty$.

(iii) Since $u_{n}^{\prime}(0)=v_{n}^{\prime}(0)=0$ and $\beta_{n}<0$,

$$
0<H_{n}(0) \leq u_{n}^{2}(0)\left[\frac{u_{n}^{2}(0)}{2}-1\right]+v_{n}^{2}(0)\left[\frac{v_{n}^{2}(0)}{2}-1\right],
$$

and hence $\max \left\{u_{n}(0), v_{n}(0)\right\}>\sqrt{2}$ for all $n$. Since $u_{n}(0) \rightarrow u(0)$ and $v_{n}(0) \rightarrow v(0)$ by (4.6), we conclude that $\max \{u(0), v(0)\} \geq \sqrt{2}$. 
Lemma 4.5. Let $0<r_{1}<r_{2}<1$.

(i) If $u \equiv 0$ on $\left[r_{1}, r_{2}\right]$, then $u_{n}^{\prime} \rightarrow 0$ uniformly on every closed interval contained in $\left(r_{1}, r_{2}\right)$.

(ii) If $v \equiv 0$ on $\left[r_{1}, r_{2}\right]$, then $v_{n}^{\prime} \rightarrow 0$ uniformly on every closed interval contained in $\left(r_{1}, r_{2}\right)$.

Proof. (i) By assumption and uniform convergence, $u_{n}<1$ on $\left[r_{1}, r_{2}\right]$ for $n$ large, hence

$$
\left(r^{N-1} u_{n}^{\prime}\right)^{\prime}=r^{N-1}\left(u_{n}-u_{n}^{3}-\beta_{n} v_{n}^{2} u_{n}\right)>0 \quad \text { on }\left[r_{1}, r_{2}\right] .
$$

For $r \in\left[r_{1}, r_{2}\right]$ we therefore have

$$
u_{n}\left(r_{2}\right)>u_{n}\left(r_{2}\right)-u_{n}(r)=\int_{r}^{r_{2}} u_{n}^{\prime}(s) d s \geq \int_{r}^{r_{2}} s^{N-1} u_{n}^{\prime}(s) d s \geq\left(r_{2}-r\right) r_{1}^{N-1} u_{n}^{\prime}(r)
$$

and

$-u_{n}\left(r_{1}\right)<u_{n}(r)-u_{n}\left(r_{1}\right)=\int_{r_{1}}^{r} u_{n}^{\prime}(s) d s \leq r_{1}^{1-N} \int_{r_{1}}^{r} s^{N-1} u_{n}^{\prime}(s) d s \leq\left(\frac{r_{2}}{r_{1}}\right)^{N-1}\left(r-r_{1}\right) u_{n}^{\prime}(r)$.

Now consider points $r_{1}<s_{1}<s_{2}<r_{2}$. Then, for every $r \in\left[s_{1}, s_{2}\right]$,

$$
-\frac{r_{1}^{N-1} u_{n}\left(r_{1}\right)}{r_{2}^{N-1}\left(s_{1}-r_{1}\right)} \leq-\frac{r_{1}^{N-1} u_{n}\left(r_{1}\right)}{r_{2}^{N-1}\left(r-r_{1}\right)} \leq u_{n}^{\prime}(r) \leq \frac{u_{n}\left(r_{2}\right)}{\left(r_{2}-r\right) r_{1}^{N-1}} \leq \frac{u_{n}\left(r_{2}\right)}{\left(r_{2}-s_{2}\right) r_{1}^{N-1}} \text {. }
$$

Consequently,

$$
\max _{\left[s_{1}, s_{2}\right]}\left|u_{n}^{\prime}\right| \leq C_{6} \max \left\{u_{n}\left(r_{1}\right), u_{n}\left(r_{2}\right)\right\} \rightarrow 0 \quad \text { as } n \rightarrow \infty .
$$

Thus (i) is true. The proof of (ii) is similar.

Next we introduce the bounded nonnegative nonincreasing function

$$
h_{\infty}:[0,1] \rightarrow \mathbb{R}, \quad h_{\infty}(r):=\liminf _{n \rightarrow \infty} H_{n}(r) \quad \text { for } 0 \leq r \leq 1 .
$$

Lemma 4.6. (i) If $N=1$, then $h_{\infty}$ equals a positive constant in $[0,1]$. (ii) If $N \geq 2$ and $\max \{u(r), v(r)\}>0$ for some $r<1$, then $h_{\infty}(r)>0$.

Proof. (i) If $N=1$, then the functions $H_{n}$ are constant by (4.2), hence $h_{\infty}$ is also constant. By integration and Lemma 4.4(ii), we get

$$
\begin{aligned}
h_{\infty}(r) & =\liminf _{n \rightarrow \infty} \int_{0}^{1} H_{n}(s) d s \\
& =\liminf _{n \rightarrow \infty} \int_{0}^{1}\left(\left|u_{n}^{\prime}\right|^{2}+\left|v_{n}^{\prime}\right|^{2}-\left(u_{n}^{2}+v_{n}^{2}\right)+\frac{1}{2}\left(u_{n}^{4}+v_{n}^{4}\right)\right) d s \\
& \geq \int_{0}^{1}\left(\left|u^{\prime}\right|^{2}+\left|v^{\prime}\right|^{2}-\left(u^{2}+v^{2}\right)+\frac{1}{2}\left(u^{4}+v^{4}\right)\right) d s=\int_{0}^{1}\left(H_{u}+H_{v}\right) d s
\end{aligned}
$$

where $H_{u}=\left|u^{\prime}\right|^{2}-u^{2}+\frac{u^{4}}{2}$ and $H_{v}=\left|v^{\prime}\right|^{2}-v^{2}+\frac{v^{4}}{2}$. Let $I \subset P(u)$ be a maximal open subinterval. Since $H_{u}^{\prime}=2 u^{\prime}\left(u^{\prime \prime}-u+u^{3}\right)=0$ in $P(u)$ by Lemma 4.1(ii), $H_{u}$ is constant in $I$. An elementary phase plane analysis shows that if $H_{u} \leq 0$ in $I$, then $u$ is bounded away from zero in $I$ (since $I$ is bounded), which contradicts the maximality 
of $I$. Hence $H_{u}>0$ in $I$, and therefore $H_{u}>0$ in $P(u)$. In the same way we deduce that $H_{v}>0$ in $P(v)$. Since $H_{u}=0$ a.e. on the zero set of $u$ and $H_{v}=0$ a.e. on the zero set of $v$, we conclude that

$$
h_{\infty}(r) \geq \int_{0}^{1}\left(H_{u}(s)+H_{w}(s)\right) d s>0,
$$

as claimed.

(ii) We may assume that $u(r)>0$. Since $H_{n}(1)=\left(u_{n}^{\prime}(1)\right)^{2}+\left(v_{n}^{\prime}(1)\right)^{2}>0,(4.2)$ implies

$$
H_{n}(r) \geq-\int_{r}^{1} H_{n}^{\prime}(s) d s=\int_{r}^{1} \frac{N-1}{s}\left[\left|u_{n}^{\prime}\right|^{2}+\left|v_{n}^{\prime}\right|^{2}\right] d s \geq \int_{r}^{1}\left|u_{n}^{\prime}\right|^{2} d s,
$$

so that by weak convergence $u_{n} \rightarrow u$ in $H^{1}(\mathbb{B})$,

$$
h_{\infty}(r) \geq \int_{r}^{1}\left|u^{\prime}\right|^{2} d s \geq \frac{1}{1-r}\left(\int_{r}^{1} u^{\prime} d s\right)^{2}=\frac{u^{2}(r)}{1-r}>0 .
$$

We now have all the tools to study the intersection properties of $u_{n}$ and $v_{n}$ resp. $u$ and $v$.

Lemma 4.7. Suppose that $0<r_{0}<1$ are such that $u\left(r_{0}\right)>0, u(r) \geq 0$ and $v(r)=0$ for $r_{0} \leq r \leq 1$. Then $u_{n} \geq v_{n}$ on $\left[r_{0}, 1\right]$ for $n$ sufficiently large.

Remark 4.8. The analoguous statement is true with the roles of $u$ and $v$ (resp. of $u_{n}$ and $v_{n}$ ) exchanged.

Proof. By uniform convergence we have $v_{n}<\min \left\{1, u\left(r_{0}\right)\right\}$ on $\left[r_{0}, 1\right]$ for $n$ large, so that $\Delta v_{n}>0$ on $\left[r_{0}, 1\right]$ and therefore

$$
v_{n}(r) \leq \max \left\{v_{n}\left(r_{0}\right), v_{n}(1)\right\}=v_{n}\left(r_{0}\right)=o\left(\left|\beta_{n}\right|^{-1}\right) \quad \text { for } r_{0} \leq r \leq 1
$$

by Lemma 4.1(i). Hence a short calculation shows that $w_{n}=u_{n}-v_{n}$ satisfies

$$
w_{n}^{3}=-\Delta w_{n}+\left[1+\left(\beta_{n}-3\right) u_{n} v_{n}\right] w_{n}=-\Delta w_{n}+[1+o(1)] w_{n} \quad \text { in }\left(r_{0}, 1\right) .
$$

Suppose by contradiction that, for a subsequence, there are points $r_{0}<r_{1}^{n}<r_{2}^{n} \leq 1$ such that $w_{n}\left(r_{1}^{n}\right)=0=w_{n}\left(r_{2}^{n}\right)$ and $w_{n}(r)<0$ for $r_{1}^{n}<r<r_{2}^{n}$. Then, multiplying (4.9) with $w_{n}$ and integrating by parts, we obtain

$$
\begin{aligned}
\int_{r_{1}^{n}}^{r_{2}^{n}} r^{N-1} w_{n}^{4} d x=\int_{r_{1}^{n}}^{r_{2}^{n}} r^{N-1}\left(\left|w_{n}^{\prime}\right|^{2}+[1+o(1)] w_{n}^{2}\right) d r & \geq \int_{r_{1}^{n}}^{r_{2}^{n}} r^{N-1}\left|w_{n}^{\prime}\right|^{2} d r \\
& \geq C_{7}\left(\int_{r_{1}^{n}}^{r_{2}^{n}} r^{N-1} w_{n}^{4} d r\right)^{\frac{1}{2}}
\end{aligned}
$$

for $n$ large, so that $\int_{r_{0}}^{1} r^{N-1}\left|w_{n}^{-}\right|^{4} d r \geq \int_{r_{1}^{n}}^{r_{2}^{n}} r^{N-1} w_{n}^{4} d r \geq C_{7}^{2}$. This however contradicts the fact that $w_{n}^{-} \rightarrow 0$ uniformly on $\left[r_{0}, 1\right]$ by assumption.

Lemma 4.9. Suppose that $0<r_{1}<r_{2}<r_{3}<1$ are such that $u\left(r_{1}\right)>0, u\left(r_{2}\right)=0$, and $u\left(r_{3}\right)>0$. Then there exists $r \in\left(r_{1}, r_{3}\right)$ with $v(r)>0$. 
Remark 4.10. Again, the analoguous statement is true with the roles of $u$ and $v$ exchanged.

Proof. By uniform convergence $u_{n} \rightarrow u$, the asumptions on $u$ imply that there exists $\varepsilon_{0}>0$ and, for large $n, \tau_{n} \in\left[r_{1}+\varepsilon_{0}, r_{3}-\varepsilon_{0}\right]$ with $u_{n}^{\prime}\left(\tau_{n}\right)=0$ and $u_{n}\left(\tau_{n}\right) \rightarrow 0$. Now suppose by contradiction that $v \equiv 0$ on $\left[r_{1}, r_{3}\right]$. Then $v_{n} \rightarrow 0$ and $v_{n}^{\prime} \rightarrow 0$ uniformly on $\left[r_{1}+\varepsilon_{0}, r_{3}-\varepsilon_{0}\right]$ by Lemma 4.5 , and therefore

$$
H_{n}\left(r_{3}\right) \leq H_{n}\left(\tau_{n}\right) \leq\left|u_{n}^{\prime}\left(\tau_{n}\right)\right|^{2}+\left|v_{n}^{\prime}\left(\tau_{n}\right)\right|^{2}+\frac{1}{2}\left(u_{n}^{4}\left(\tau_{n}\right)+v_{n}^{4}\left(\tau_{n}\right)\right)=o(1) .
$$

This contradicts Lemma 4.6. Hence there exists $r \in\left(r_{1}, r_{3}\right)$ with $v(r)>0$.

Lemma 4.11. Suppose that $0<r_{1}<r_{2}<r_{3}<1$ are such that $u\left(r_{1}\right)>0, v\left(r_{3}\right)>0$, $v \equiv 0$ in $\left[r_{1}, r_{2}\right]$ and $u \equiv 0$ in $\left[r_{2}, r_{3}\right]$. Then, for $n$ sufficiently large, $u_{n}-v_{n}$ has precisely one zero in $\left(r_{1}, r_{3}\right)$.

Remark 4.12. Again, the analoguous statement is true with the roles of $u$ and $v$ (resp. of $u_{n}$ and $v_{n}$ ) exchanged.

Proof. Since $h_{\infty}\left(r_{3}\right)>0$ by Lemma 4.6 , we may choose $0<\varepsilon<\min \left\{1, u\left(r_{1}\right), v\left(r_{3}\right)\right\}$ such that

$$
\varepsilon^{4}+2 \varepsilon^{2}<h_{\infty}\left(r_{3}\right) .
$$

Let $s_{1} \in\left(r_{1}, r_{2}\right], s_{2} \in\left[r_{2}, r_{3}\right)$ be such that

$$
u\left(s_{1}\right)=\varepsilon, u(r)<\varepsilon \text { for } s_{1}<r \leq r_{3} \quad \text { and } \quad v\left(s_{2}\right)=\varepsilon, v(r)<\varepsilon \text { for } r_{1} \leq r<s_{2} .
$$

By assumption and Lemma 4.9 we have $u>0$ on $\left[r_{1}, s_{1}\right]$ and $v>0$ on $\left[s_{2}, r_{3}\right]$. Thus $s_{1}<s_{2}$ and

$$
v_{n}<u_{n} \quad \text { on }\left[r_{1}, s_{1}\right], \quad u_{n}<v_{n} \quad \text { on }\left[s_{2}, r_{3}\right] \quad \text { for } n \text { large. }
$$

Since, by Lemma 4.4(i), $v \equiv 0$ in a neighborhood of $r_{1}$ and $u \equiv 0$ in a neighborhood of $r_{3}$, Lemma 4.5 implies that

$$
u_{n}^{\prime}\left(r_{3}\right)<\left(\frac{s_{1}}{r_{3}}\right)^{N-1} \varepsilon \quad \text { and } \quad v_{n}^{\prime}\left(r_{1}\right)>-\varepsilon \quad \text { for } n \text { large. }
$$

For $n$ large we also have $u_{n}<1$ on $\left[s_{1}, r_{3}\right]$, therefore

$$
\left(r^{N-1} u_{n}^{\prime}\right)^{\prime}=r^{N-1} \Delta u_{n}>0,
$$

so that $r^{N-1} u_{n}^{\prime}$ is increasing in $\left[s_{1}, r_{3}\right]$. Similarly, $r^{N-1} v_{n}^{\prime}$ is increasing in $\left[r_{1}, s_{2}\right]$. So (4.12) implies that

$$
u_{n}^{\prime}<\varepsilon \text { on }\left[s_{1}, r_{3}\right] \text { and } v_{n}^{\prime}>-\varepsilon \text { on }\left[r_{1}, s_{2}\right] \quad \text { for } n \text { large. }
$$

Now suppose by contradiction that, for a subsequence, the functions $u_{n}-v_{n}$ have at least two zeros in $\left(r_{1}, r_{3}\right)$. By (4.11) these points must lie in $\left(s_{1}, s_{2}\right)$ for large $n$. Hence 
there is a point $\tau_{n} \in\left(s_{1}, s_{2}\right)$ with $u_{n}^{\prime}\left(\tau_{n}\right)=v_{n}^{\prime}\left(\tau_{n}\right)$, so that $\left|u_{n}^{\prime}\left(\tau_{n}\right)\right|=\left|v_{n}^{\prime}\left(\tau_{n}\right)\right|<\varepsilon$ by (4.13). Hence

$$
\begin{aligned}
H_{n}\left(\tau_{n}\right) & \leq\left|u_{n}^{\prime}\left(\tau_{n}\right)\right|^{2}+\left|v_{n}\left(\tau_{n}\right)^{\prime}\right|^{2}+\frac{1}{2}\left(u_{n}^{4}\left(\tau_{n}\right)+v_{n}^{4}\left(\tau_{n}\right)\right) \\
& \leq 2 \varepsilon^{2}+\varepsilon^{4}+o(1)
\end{aligned}
$$

We conclude that

$$
h_{\infty}\left(r_{3}\right)=\liminf _{n \rightarrow \infty} H_{n}\left(r_{3}\right) \leq \liminf _{n \rightarrow \infty} H_{n}\left(\tau_{n}\right) \leq 2 \varepsilon^{2}+\varepsilon^{4},
$$

which contradicts (4.10). The proof is finished.

Corollary 4.13. The function $w=u-v$ is a radial solution of (1.4) with $E_{S}(w)=c_{k}$ which has precisely $k-1$ interior zeros. Moreover, $u_{n} \rightarrow u$ and $v_{n} \rightarrow v$ in $H^{1}(\mathbb{B})$.

Proof. Since $w_{n}:=u_{n}-v_{n}$ changes sign precisely $k-1$ times in $(0,1)$ for every $n$ and $w_{n} \rightarrow w$ uniformly in $[0,1]$, the function $w$ changes sign at most $k-1$ times. On the other hand, since $u \cdot v=0$ in $[0,1]$, Lemma 4.11 implies that in every subinterval where $w$ changes sign precisely once, $w_{n}$ also changes sign precisely once for large $n$. Together with Lemmas 4.4(iii), 4.7 and 4.9 this implies that $w$ changes sign precisely $k-1$ times in $[0,1]$. Moreover, by weak convergence and Lemma 4.4(ii),

$$
\begin{aligned}
E_{S}(w) & =E_{S}(u)+E_{S}(v) \leq \liminf _{n \rightarrow \infty}\left(E_{S}\left(u_{n}\right)+E_{S}\left(v_{n}\right)\right) \\
& =\liminf _{n \rightarrow \infty}\left(E\left(u_{n}, v_{n}\right)+\frac{\beta}{2} \int_{\mathbb{B}} u_{n}^{2} v_{n}^{2}\right)=\liminf _{n \rightarrow \infty} E\left(u_{n}, v_{n}\right) \leq c_{k} .
\end{aligned}
$$

Corollary 4.2 implies that $w$ is contained in the set $\Gamma_{k}$ defined in Section 2, so that $w$ is a minimizer of the minimization problem $(2.2)$. Thus $E_{S}(w)=c_{k}$, and $w$ is a radial solution of (1.4) having precisely $k-1$ interior zeros by Proposition 2.1. A posteriori we conclude that equality holds in all steps in (4.15), and therefore

$$
\int_{\mathbb{B}}\left|\nabla u_{n}\right|^{2} d x \rightarrow \int_{\mathbb{B}}|\nabla u|^{2} d x, \quad \int_{\mathbb{B}}\left|\nabla v_{n}\right|^{2} d x \rightarrow \int_{\mathbb{B}}|\nabla v|^{2} d x \quad \text { as } n \rightarrow \infty .
$$

Hence $u_{n} \rightarrow u$ and $v_{n} \rightarrow v$ in $H^{1}(\mathbb{B})$, as claimed.

Theorem 1.2 is a direct consequence of (4.6), Lemma 4.4(i) and Corollary 4.13.

\section{REFERENCES}

[1] H. Amann. Global existence for semilinear parabolic systems. J. Reine Angew. Math., 360:47-83, 1985.

[2] A. Ambrosetti and E. Colorado. Bound and ground states of coupled nonlinear Schrodinger equations. C.R. Math. Acad. Sci. Paris, 342:453-458, 2006.

[3] N. Akhmediev and A. Ankiewicz. Partially coherent solitons on a finite background. Phys. Rev. Lett., 82:2661-2665, 1998.

[4] S. Angenent. The zero set of a solution of a parabolic equation. J. Reine Angew. Math., 390:79-96, 1988.

[5] T. Bartsch, Z.-Q. Wang and J. Wei. Bound states for a coupled Schrödinger system. preprint.

[6] T. Bartsch and M. Willem. Infinitely many radial solutions of a semilinear elliptic problem on $\mathbf{R}^{N}$. Arch. Rational Mech. Anal., 124(3):261-276, 1993. 
[7] T. Cazenave and P.-L. Lions. Solutions globales d'équations de la chaleur semi linéaires. Comm. Partial Differential Equations, 9(10):955-978, 1984.

[8] S. Chang, C.S. Lin, T.C. Lin and W. Lin. Segregated nodal domains of two-dimensional multispecies Bose-Einstein condensates. Phys. D , 196(3-4):341-361, 2004.

[9] D. N. Christodoulides, T. H. Coskun, M. Mitchell and M. Segev. Theory of incoherent self-focusing in biased photorefractive media. Phys. Rev. Lett., 78:646-649, 1997.

[10] M. Conti, L. Merizzi and S. Terracini. Radial solutions of superlinear equations on $R^{N}$. I. A global variational approach. Arch. Ration. Mech. Anal., 153(4):291-316, 2000.

[11] M. Conti and S. Terracini. Radial solutions of superlinear equations on $R^{N}$. II. The forced case. Arch. Ration. Mech. Anal., 153(4):317-339, 2000.

[12] M. Conti, S. Terracini and G. Verzini. Nehari's problem and competing species systems. Ann. Inst. H. Poincaré Anal. Non Linéaire, 19(6):871-888, 2002.

[13] M. Conti, S. Terracini and G. Verzini. Asymptotic estimates for the spatial segregation of competitive systems. Adv. Math., 195(2):524-560, 2005.

[14] E.N. Dancer and Y. Du. Competing species equations with diffusion, large interactions, and jumping nonlinearities. J. Differential Equations, 114(2):434-475, 1994.

[15] B. D. Esry, C. H. Greene, J. P. Burke Jr., J. L. Bohn. Hartree-Fock theory for double condensates. Phys. Rev. Lett., 78:3594-3597, 1997.

[16] D. Gilbarg and N. S. Trudinger. Elliptic Partial Differential Equations of Second order, 2nd.ed, Springer-Verlag, 1983.

[17] D.S. Hall, M.R. Matthews, J.R. Ensher, C.E. Wieman, E.A. Cornell. Dynamics of component separation in a binary mixture of Bose-Einstein condensates. Phys. Rev. Lett., 81:1539-1542, 1998.

[18] D.B. Henry. Some infinite-dimensional Morse-Smale systems defined by parabolic partial differential equations. J. Differential Equations, 59:165-205, 1985.

[19] F. T. Hioe, Solitary Waves for N Coupled Nonlinear Schrödinger Equations. Phys. Rev. Lett., 82:1152-1155, 1999.

[20] F. T. Hioe, T. S. Salter. Special set and solutions of coupled nonlinear Schrödinger equations. J. Phys. A: Math. Gen., 35:8913-8928, 2002.

[21] T. Kanna and M. Lakshmanan. Exact soliton solutions, shape changing collisions, and partially coherent solitons in coupled nonlinear Schrödinger equations. Phys. Rev. Lett., 86:5043-5046, 2001.

[22] T.-C. Lin and J.-C. Wei. Ground state of $N$ coupled Nonlinear Schrödinger Equations in $R^{n}$, $n \leq 3$. Communications in Mathematical Physics, 255(3):629-653, 2005.

[23] T. C. Lin and J. Wei. Spikes in two coupled nonlinear Schrödinger equations, Ann. Inst. H. Poincaré Anal. Non Linéaire, 22(4):403-439, 2005.

[24] T. C. Lin and J. Wei. Solitary and self-similar solutions of two-component system of nonlinear Schrödinger equations. Physica D: Nonlinear Phenomena, to appear.

[25] L.A. Maia, E. Montefusco and B. Pellacci. Positive solutions for a weakly coupled nonlinear Schrödinger system. J. Differential Equations, 229:743-767, 2006.

[26] H. Matano. Nonincrease of the lap-number of a solution for a one-dimensional semilinear parabolic equation. J. Fac. Sci. Univ. Tokyo Sect. IA Math., 29:401-441, 1982.

[27] M. Mitchell, Z. Chen, M. Shih, and M. Segev. Self-Trapping of partially spatially incoherent light. Phys. Rev. Lett., 77:490-493, 1996.

[28] M. Mitchell and M. Segev. Self-trapping of incoherent white light. Nature, 387:880-882, 1997.

[29] C.J. Myatt, E.A. Burt, R.W. Ghrist, E.A. Cornell, C.E. Wieman. Production of two overlapping Bose-Einstein condensates by sympathetic cooling. Phys. Rev. Lett., 78:586-589, 1997.

[30] Z. Nehari. Characteristic values associated with a class of non-linear second-order differential equations. Acta Math., 105:141-175, 1961.

[31] K. Nickel. Gestaltaussagen über Lösungen parabolischer Differentialgleichungen. J. Reine Angew. Math., 211:78-94, 1962. 
[32] X.Y. Chen and P. Poláčik. Asymptotic periodicity of positive solutions of reaction diffusion equations on a ball, J. Reine Angew. Math., 472:17-51, 1996.

[33] P. Quittner. Multiple equilibria, periodic solutions and a priori bounds for solutions in superlinear parabolic problems. NoDEA Nonlinear Differential Equations Appl., 11:237-258, 2004.

[34] D. H. Sattinger. On the total variation of solutions of parabolic equations. Math. Ann., 183:7892, 1969.

[35] B. Sirakov. Least energy solitary waves for a system of nonlinear Schrödinger equations. preprint.

[36] S. Terracini and G. Verzini. Solutions of prescribed number of zeroes to a class of superlinear ODE's systems. NoDEA Nonlinear Differential Equations Appl., 8:323-341, 2001.

[37] E. Timmermans. Phase separation of Bose-Einstein condensates. Phys. Rev. Lett., 81:5718-5721, 1998.

[38] W. C. Troy. Symmetry properties in systems of semilinear elliptic equations. J. Differential Equations, 42(3):400-413, 1981.

[39] M. Struwe. Multiple solutions of anticoercive boundary value problems for a class of ordinary differential equations of second order. J. Differential Equations, 37(2):285-295, 1980.

[40] M. Struwe. Infinitely many solutions of superlinear boundary value problems with rotational symmetry. Arch. Math. (Basel), 36(4):360-369, 1981.

[41] M. Struwe. Superlinear elliptic boundary value problems with rotational symmetry. Arch. Math. (Basel), 39(3):233-240, 1982.

[42] M. Struwe. Variational Methods. Springer, Berlin-Heidelberg 1990.

[43] J.C. Wei, T. Weth. Nonradial symmetric bound states for a system of coupled Schrödinger equations. preprint.

[44] M. Willem. Minimax theorems. PNLDE 24, Birkhäuser, Boston-Basel-Berlin 1996.

Department of Mathematics, Chinese University of Hong Kong, Shatin, Hong Kong E-mail address: wei@math.cuhk.edu.hk

Mathematische Institut, Universitat Giessen, Arndtstr.2, 35392 Giessen, Germany

E-mail address: tobias.weth@math.uni-giessen.de 